\title{
Triggered star formation in bright-rimmed clouds
}

\author{
L. K. Morgan $^{1} \dagger$, J. S. Urquhart ${ }^{1,2}$, M. A. Thompson ${ }^{1,3}$ \\ and G. J. White ${ }^{1,4,5}$ \\ ${ }^{1}$ CAPS, The University Of Kent, Canterbury, Kent CT2 7NR \\ ${ }^{2}$ University Of Leeds (UK) \\ ${ }^{3}$ The University Of Hertfordshire (UK) \\ ${ }^{4}$ CCLRC Rutherford Appleton Laboratory (UK) \\ ${ }^{5}$ The Open University (UK)
}

\begin{abstract}
A sample of optically Bright-Rimmed Clouds (BRC) at the edge of HII regions has been observed at multiple wavelengths in order to investigate the possibility that star-formation is present. Such activity may be related to photoionisation induced shocks caused by the massive stars powering the HII regions.

The sample has been observed at radio, infrared and submillimetre wavelengths. Both molecular line studies and continuum observations have been made of the larger cloud structures and embedded sources within.

Radio and infrared continuum observations show the presence of ionised boundary layers coincident with the optically bright rims. These are responsible for the propagation of shocks into the clouds interiors, possibly triggering the collapse of cores into protostars.

Molecular line studies and submillimetre continuum observations show the presence of centrally condensed cores within the clouds, these cores have high densities and have submillimetre luminosities indicative of class 0 protostars. The total luminosities of the embedded sources reveal a set of forming intermediate to high-mass stars.

The identification of these regions as star-forming has important consequences for studies of triggered star-formation, not only does the high incidence of star formation in BRC suggest a high efficiency for Galactic triggered star-formation but the masses of the sources suggest a preferred process for the formation itself.
\end{abstract}

$\dagger$ Present address: Green Bank Observatory, P.O.Box 2, Green Bank, WV 24944 UNITED STATES 